\title{
PECTINATELLA MAGNIFICA LEIDY 1851 (PHYLACTOLAEMATES), UN BRYOZOAIRE INTRODUIT DANS LE NORD FRANCHE-COMTÉ.
}

\author{
S. RODRIGUEZ (1), J.P. VERGON (2)
}

(1) Bureau d'études Sylvaine Rodriguez Hydrobiologie - Hydroécologie, 25 rue Pierre et Marie Curie, 90300 CRAVANCHE, France.

(2) Direction Régionale de l'Environnement de Franche-Comté, Service de l'Eau et des Milieux Naturels, cellule gestion des milieux aquatiques, 5 rue du Général Sarrail, B.P. 137, 25014 BESANÇON Cedex, France.

\section{RÉSUMÉ}

A partir de l'observation initiale d'une colonie du bryozoaire Pectinatella magnifica Leidy 1851 dans le nord Franche-Comté (été 1994) et d'une analyse bibliographique, un suivi de l'extension de l'espèce a été effectué sur le territoire franc-comtois et dans les régions limitrophes. La localisation des stations est assortie de valeurs de qualité d'eau mesurées sur le réseau hydrographique franc-comtois pour le compte de l'Agence de l'Eau Rhône-Méditerranée-Corse. La rédaction d'une fiche d'enquête nationale vise à collecter des informations sur ce bryozoaire afin d'en faciliter ultérieurement la gestion.

Mots-clés: Pectinatella magnifica, Bryozoaires, Phylactolaemates, eau douce, Franche-Comté, France, écologie, répartition.

\section{PECTINATELLA MAGNIFICA LEIDY 1851 (PHYLACTOLAEMATES), A SPECIES OF BRYOZOA INTRODUCED IN THE NORTH OF FRANCHE-COMTÉ.}

\section{ABSTRACT}

Following the first mention of a colony of the bryozoa Pectinatella magnifica in northern Franche-Comté (summer 1994) and a bibliographic investigation, the spread of the species was followed in Franche-Comté and neighbouring regions. The geographic location of the stations is completed with water quality data collected from the hydrographic basins of Franche-Comté by the RMC Agency. The compiling of a national enquiry form is directed to the collecting of information on this bryozoa for its future management.

Key-words : Pectinella magnifica, Bryozoa, Phylactoleamates, freshwater, FrancheComté, France, ecology, spread. 


\section{INTRODUCTION}

Le nord-est de la Franche-Comté et la marge occidentale de l'Ajoie, en territoire helvétique, occupent l'ancien passage du lien fluvial entre les bassins du Rhin et du Rhône et constituent un lieu privilégié d'échanges faunistiques entre les deux zones biogéographiques.

L'extension de l'aire de répartition d'espèces aquatiques, rares pour la FrancheComté, dans le territoire de Belfort et le pays de Montbéliard en témoignent: Craspedacusta sowerbyi Cnidaires (ALOCAT, 1961) et Misgurnus fossilis Cobitidae (communication orale BOULANGER D., CSP Territoire de Belfort et MONCORGÉ S., Espace Naturel Comtois).

Pectinatella magnifica Leidy 1851 (Phylactolaemates) confirme cette particularité. Sporadique en 1994, l'espèce semble s'étendre à un nombre élargi de plans d'eau et de cours d'eau de Franche-Comté et des régions limitrophes. Cette extension occidentale récemment accrue (1999) permet de formuler l'hypothèse d'une distribution des statoblastes (petites capsules constituant des formes de résistance), liée au transport particulaire de haute altitude résultant d'épisodes climatiques particuliers (tempêtes, tornades), aux côtés de modes de propagation plus connus. Les propriétaires de plans d'eau et les acteurs de la pêche s'inquiètent de l'envahissement massif des eaux de surface par des colonies très difficiles à éradiquer.

\section{CIRCONSTANCES}

Au cours de l'été 1994, le personnel du Service des voies navigables de France recueillait sur le canal de Haute-Saône, à la hauteur de Bermont (Territoire de Belfort Franche-Comté), une colonie gélatineuse ovoïde de diamètre voisin du mètre, d'un volume proche de cent litres et l'acheminait à la Direction Régionale de l'Environnement (DIREN de Franche-Comté), afin d'en connaître la position taxinomique et l'influence sur le milieu aquatique.

Classée en premier examen parmi les bryozoaires phylactolaemates, à partir de l'ouvrage de TACHET et al., 1991, la colonie s'apparentait à la famille des Cristatellidae. Sa taille et la forme des épines des statoblastes différents de ceux de l'espèce qui semblait la plus proche (Cristatella mucedo Cuvier, 1798) ont conduit à préciser la position systématique de l'échantillon.

Préalablement à cette recherche, un fragment de colonie avait été conservé (formol à $5 \%$ de concentration finale) dans les collections de la DIREN de Franche-Comté et du bureau d'études Sylvaine Rodriguez.

Au début de l'automne 1998, dans le cadre d'un inventaire des Bryozoaires d'eaux douces et de recherche d'une espèce récemment apparue en France, Pectinatella magnifica Leidy 1851, NOTTENGHEM et D'HONDT, porteurs du programme, avaient été destinataires d'un échantillon franc-comtois de 1994, pour identification, confirmée au Muséum National d'Histoire Naturelle de Paris par J.L. D’HONDT.

Cette observation, non publiée à l'époque, est la plus ancienne de France, d'autres mentions étant intervenues depuis sur le territoire franc-comtois et dans d'autres régions (D'HONDT et CONDÉ, 1996 ; NOTTENGHEM, 1999). 


\section{MORPHOLOGIE DES COLONIES}

Les colonies de Pectinatella magnifica développées sur des supports allongés (branches) forment une sorte de manchon (NOTTENGHEM, 1999) atteignant parfois $1 \mathrm{~m}$ de long (PRENANT et BOBIN, 1956). Sur des surfaces larges et planes, P. magnifica croît en coussinets ou en amas sphériques (PRENANT et BOBIN, 1956 ; NOTTENGHEM, 1999). PARIS (communication personnelle) signale, en 1999, la présence de colonies en forme de bouées ou d'anneaux, échouées en bordure de l'étang du Goulot (commune de Lormes, Nièvre).

Les mentions de la littérature font état de colonies de la grosseur d'une tête d'enfant (PRENANT et BOBIN, 1956) ou d'adulte (BUSHNELL, 1965). LEYDI (in JULLIEN, 1885) mentionne la récolte de "colonies gigantesques» par le $\mathrm{Dr}$ W.M. SPILLMAN ( 38 × 30 centimètres), dans des lacs de Colombus (Mississipi, USA). En France, les tailles signalées atteignent $70 \mathrm{~cm}$ (Lac de la Sorme, NOTTENGHEM, 1999), $75 \mathrm{~cm}$ (étang du Goulot, PARIS, comm. pers.) voire plus de 1 mètre de diamètre (Canal de Haute-Saône en juillet 1994 et étang sur la commune de La-chapelle-sous-Chaux (90), communication orale, Mme CHEVALIER).

Les spécimens francs-comtois forment des colonies gélatineuses de quelques millimètres (dans la Saône) à plus d'1 mètre de diamètre (Territoire de Belfort). Le plus souvent elles ont la taille et la forme d'un ballon de rugby (Figure 1).

La colonie, indifférente au support (ouvrages de maçonnerie, branchages ou végétaux aquatiques), est fixée dans son jeune âge et peut ensuite flotter librement.

A la surface de la masse gélatineuse et hyaline, les zoécies, beiges à jaune clair, sont disposées en rosette dont la taille avoisine $1 \mathrm{~cm}$ de diamètre (Figure 2).

Chaque zoécie se compose d'une sorte de logette (le cystide) où s'abrite un polype qui peut s'invaginer dans le cystide ou épanouir à l'extérieur son lophophore, composé d'une couronne de tentacules ciliés, disposés en fer à cheval (Figure 3).

A faible grossissement (10x), il est possible d'observer dans les premiers millimètres de la couche gélatineuse de petites capsules noirâtres : les statoblastes, localisés dans les cystides (Figure $4-15 \mathrm{x}$ ) et dont le diamètre varie entre 1,05 et $1,395 \mathrm{~mm}$.

Chaque statoblaste est formée d'une capsule à deux valves chitineuses accolées, sub-circulaires, brun-noir et d'un diamètre de 0,9 à 1,035 mm.

Autour de cet ensemble sombre, une couronne plus claire et clairement identifiable (Figure 5) forme l'anneau pneumatique (BRIEN, 1960) qui permet au statoblaste de flotter à l'état libre.

Sur ce flotteur s'insère, en général, une rangée de 14 épines de 150 à $285 \mu \mathrm{m}$, à extrémité distale bifide (Figure 6).

La forme, le nombre et la terminaison bifide des épines permettent, sans conteste, de ranger le bryozoaire découvert dans le canal de Haute-Saône, en juillet 1994, au sein de l'espèce Pectinatella magnifica Leidy, 1851, telle qu'elle est décrite dans la monographie des Bryozaires de JULLIEN (1885) où est retranscrite la description de l'espèce type de Leidy.

Lors du curage du canal de Haute-Saône (7-15 octobre 1998), une reconnaissance conduite par R. GAMBERI, C. GAJOT et S. RODRIGUEZ a confirmé la présence de Pectinatella magnifica au droit du pont de Brognard (Montbéliard, 25) où, sur une surface 
d'environ $60 \mathrm{~m}^{2}, 27$ colonies ovoïdes, échouées (Figure 7), présentaient une taille individuelle de 5 à $25 \mathrm{~cm}$ pour une épaisseur de 4 à $9,5 \mathrm{~cm}$.

La chronologie d'apparition des colonies, leur localisation géographique, les substrats d'accueil ainsi que l'origine des observations sont rassemblés dans le Tableau I.

\section{Tableau I}

Mentions françaises de Pectinatella magnifica Leidy 1851.

\section{Table I}

French observations of Pectinatella magnifica Leidy 1851.

\begin{tabular}{|c|c|c|c|}
\hline Date & Lieu & $\begin{array}{c}\text { Taille des } \\
\text { colonies } \\
(\mathrm{cm}) \\
\end{array}$ & $\begin{array}{l}\text { OBSERVATEURS } \\
\text { DÉTERMINATION } \\
\text { OU RÉFÉRENCES }\end{array}$ \\
\hline 7 juillet 1994 & Canal de Haute-Saône (Bermont, 90) & 100 & \\
\hline 1995 & $\begin{array}{l}\text { Etang de la Héronnière, Nomexy (88) - bassin versant } \\
\text { de la Moselle }\end{array}$ & & J.L. D’HONDT, B. CONDÉ, 1996 \\
\hline 1995 & Moselle, Châtel-sur-Moselle (88) & & P. NOTTENGHEM, 1999 \\
\hline $\begin{array}{l}\text { Fin } 96- \\
\text { début } 97\end{array}$ & Coney, Lemagny (70-88) & $>30 \times 30$ & P. MAZUER \\
\hline 1997 & $\begin{array}{l}\text { Etang sur le cours d'eau Le Monseigneur, Brû (88)- } \\
\text { bassin versant de la Mortagne affluent de la Meurthe }\end{array}$ & & P. NOTTENGHEM, 1999 \\
\hline 2 juillet 1998 & Saône (Apremont, 70) & \begin{tabular}{|c|} 
Amas de \\
statoblastes
\end{tabular} & $\begin{array}{l}\text { J.L. FALCONNET } \\
\text { S. RODRIGUEZ }\end{array}$ \\
\hline 2 juillet 1998 & Saône (Scey-sur-Saône, 70) & \begin{tabular}{|c|} 
Amas de \\
statoblastes
\end{tabular} & $\begin{array}{l}\text { J.L. FALCONNET } \\
\text { S. RODRIGUEZ }\end{array}$ \\
\hline 16 août 1998 & Saône (Scey-sur-Saône, 70) & $0,3 \times 0,8$ & $\begin{array}{c}\text { J.L. FALCONNET } \\
\text { S. RODRIGUEZ } \\
\end{array}$ \\
\hline 7 octobre 1998 & canal de Haute-Saône (Montbéliard, 25) & $25 \times 9,5$ & $\begin{array}{l}\text { S. RODRIGUEZ, R. GAMBÉRI, } \\
\text { C. GAJOT }\end{array}$ \\
\hline 1998 & Saône, Auxonne (21) - Tournus (71) & & P. NOTTENGHEM, 1999 \\
\hline 1998 & Saône, Gray (70) & & P. NOTTENGHEM, 1999 \\
\hline 1998 & Ay, Sarras (07) - bassin versant du Rhône & & P. NOTTENGHEM, 1999 \\
\hline 1998 & $\begin{array}{l}\text { Etang de la Boutelière, Vivans (42) - bassin versant de } \\
\text { La Teysonne affluent de la Loire }\end{array}$ & & P. NOTTENGHEM, 1999 \\
\hline 1998 & $\begin{array}{l}\text { Lac de Sorme, Blanzy, les Bizots et Charmoy (71), } \\
\text { bassin versant de la Bourbince affluent de la Loire }\end{array}$ & & P. NOTTENGHEM, 1999 \\
\hline 1998 & $\begin{array}{l}\text { Etang de Montheuland, Blanzy (71) - bassin versant de } \\
\text { la Loire }\end{array}$ & & P. NOTTENGHEM, 1999 \\
\hline 1998 & Etang de la Garrenne, Saint-Valier (71) & & P. NOTTENGHEM, 1999 \\
\hline Eté 1998 & Loire, Nevers (58) Lieu dit lle St-Charles & 5 à 15 & C. HEINTZ \\
\hline Eté 98 & Etang des Trémeurs, Bains-les-Bains (88) & & M. LEPETITPAS \\
\hline 1999 & Etang d'Esboz-Brest (70) - vallée du Breuchin & 20 & P. COLIN, J.P. VERGON \\
\hline 1999 & $\begin{array}{l}\text { Etang, Housseras (88) - bassin versant de la Mortagne } \\
\text { affluent de la Meurthe }\end{array}$ & & P. NOTTENGHEM, 1999 \\
\hline 16 août 1999 & Etang (Lachapelle-sous-Chaux, 90) & $>100$ & $\begin{array}{l}\text { S. RODRIGUEZ } \\
\text { J.P. VERGON }\end{array}$ \\
\hline 21 août1999 & Etang du Malsaucy (90) & 20 & S. RODRIGUEZ \\
\hline août-oct. 1999 & Etang du Goulot, Lormes (58) & $10 \times 75$ & L. PARIS \\
\hline août-oct. 1999 & $\begin{array}{l}\text { Etang de la Boue, Remilly (58) - bassin versant de la } \\
\text { Loire }\end{array}$ & 5 à 40 & C. HEINTZ \\
\hline 13 sept. 1999 & Centrale nucléaire de Belleville-sur-Loire (18) & 10 à 12 & M. KHALANSKI \\
\hline octobre 1999 & Loire, Nevers (58) Lieu dit lle St-Charles & 5 à 15 & C. HEINTZ \\
\hline 20 juin 2000 & Ognon, Palise (25) et Cromary (70) & $15 \times 15$ & $\begin{array}{l}\text { P. COMPAGNAT } \\
\text { S. RODRIGUEZ }\end{array}$ \\
\hline 25 juillet 2000 & Balastière de Choix, Lure (70) & $\begin{array}{l}\text { Fixée sur } \\
\text { branche }\end{array}$ & C. GIRARD, P. BARAN, C. GAJOT \\
\hline Eté 2000 & Isthme entre les deux lacs de Macclu (39) & & A. SHWARTZ \\
\hline
\end{tabular}




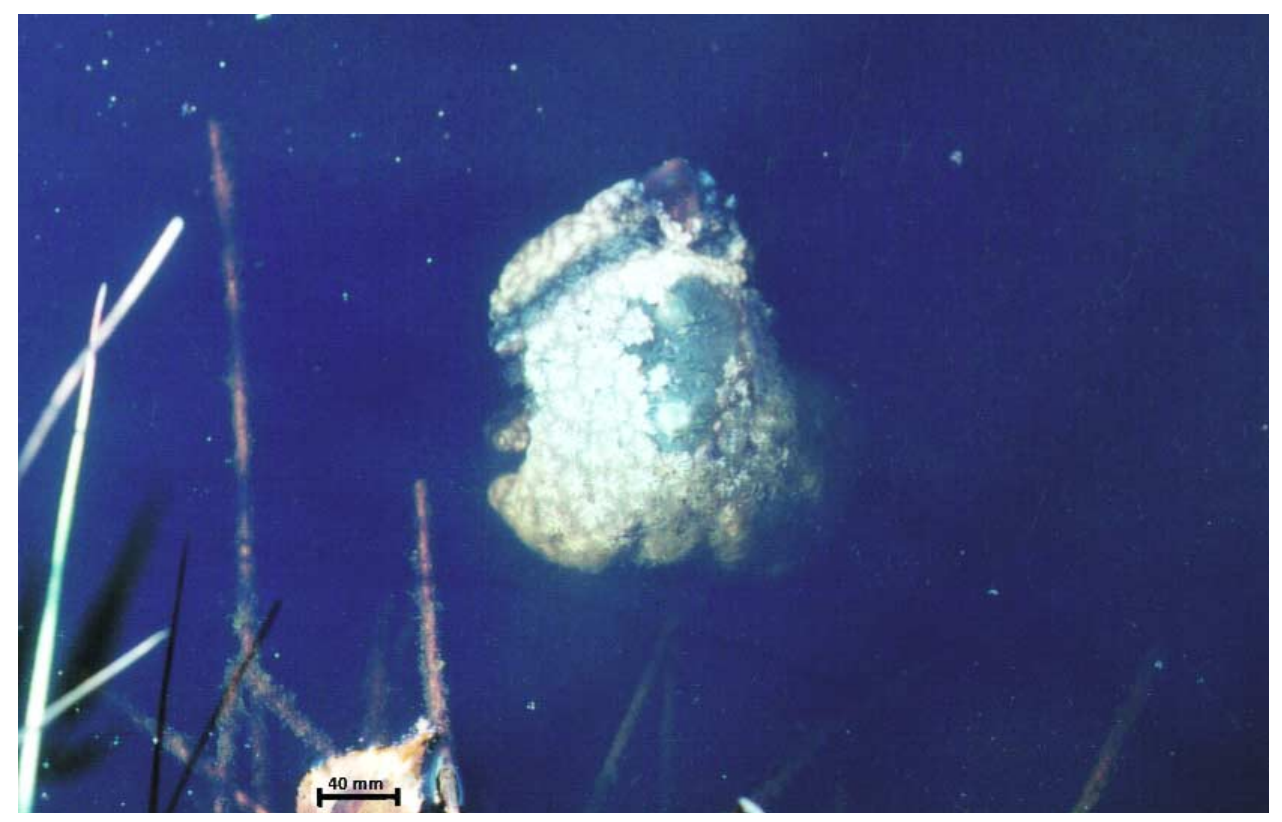

Figure 1

Colonie flottant à la surface de l'étang d'Esboz-Brest (département de la Haute-Saône) en 1999, Vallée du Breuchin sous-affluent de la Saône. Photographie de P. COLIN.

Figure 1

Floating colony on the surface on the Esboz-Brest pond (Department of Haute-Saône) in 1999, Breuchin River valley, tributary of the Saône River. Photography by P. COLIN.

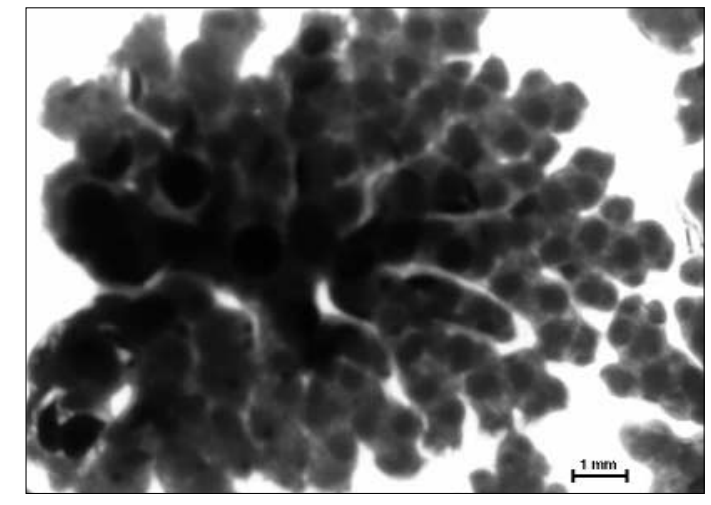

Figure 2

Zoécies disposées en rosette de P. magnifica - Canal de Haute-saône, Bermont (Territoire de Belfort - 90), 1994. Photographie de S. RODRIGUEZ.

\section{Figure 2}

Zooids of $\boldsymbol{P}$. magnifica arranged in rosette, canal of Haute-Saône, Bermont (Territoire de Belfort - 90), 1994. Photography by S. RODRIGUEZ.

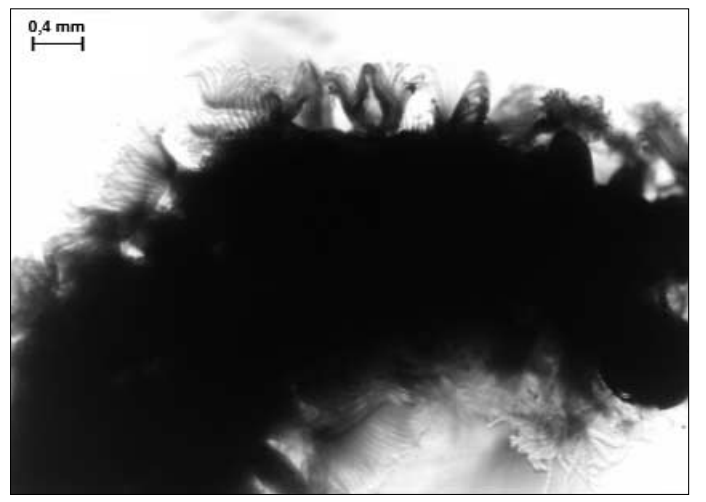

Figure 3

Lophophores d'une jeune colonie de $P$. magnifica, La Saône à Saint-Albin (département de la Haute-Saône - 70), 1998. Photographie de S. RODRIGUEZ.

Figure 3

Lophophores of a young colony of $P$. magnifica, Saône River in Saint-Albin (Department of Haute-Saône - 70), 1998. Photography by S. RODRIGUEZ. 


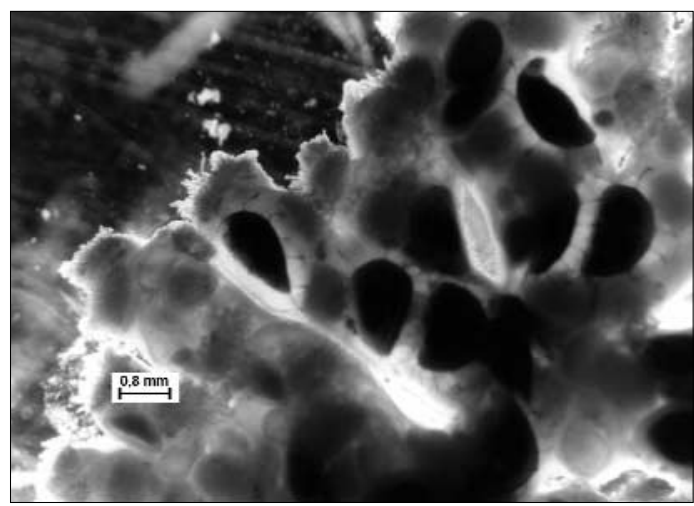

Figure 4

Cystides renfermant des statoblastes en formation, Canal de Haute-Saône, Bermont (Territoire de Belfort - 90), 1994. Photographie de S. RODRIGUEZ.

Figure 4

Cystids with forming floatobalsts, canal of Haute-Saône, Bermont (Territoire de Belfort - 90), 1994. Photography by S. RODRIGUEZ.

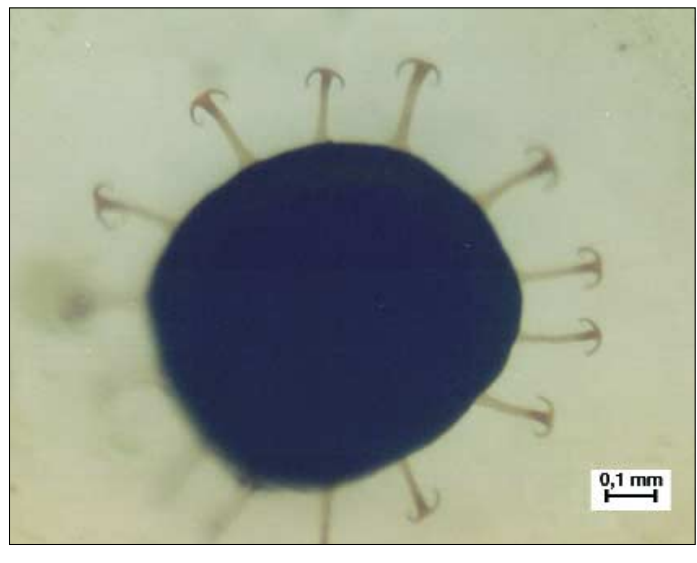

Figure 6

Statoblaste et sa couronne d'épines à extrémité bifide, Canal de Haute-Saône, Bermont (Territoire de Belfort - 90), 1998. Photographie de S. RODRIGUEZ.

Figure 6

Floatoblast and its marginal hooked spines, canal of Haute-Saône, Bermont (Territoire de Belfort - 90), 1998. Photography by S. RODRIGUEZ.

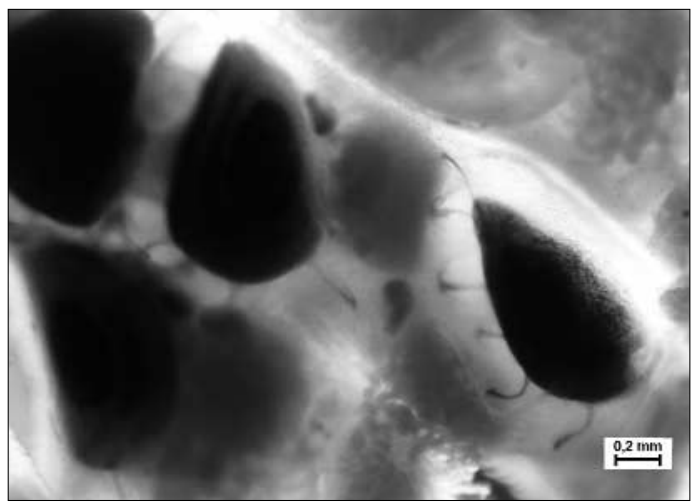

Figure 5

Statoblastes à l'intérieur de cystides, anneau pneumatique bien visible, Canal de Haute-Saône, Bermont (Territoire de Belfort - 90), 1994. Photographie de S. RODRIGUEZ.

Figure 5

Floatoblasts inside cystids with conspicuous annulus, canal of HauteSaône, Bermont (Territoire de Belfort - 90), 1994. Photography by S. RODRIGUEZ.

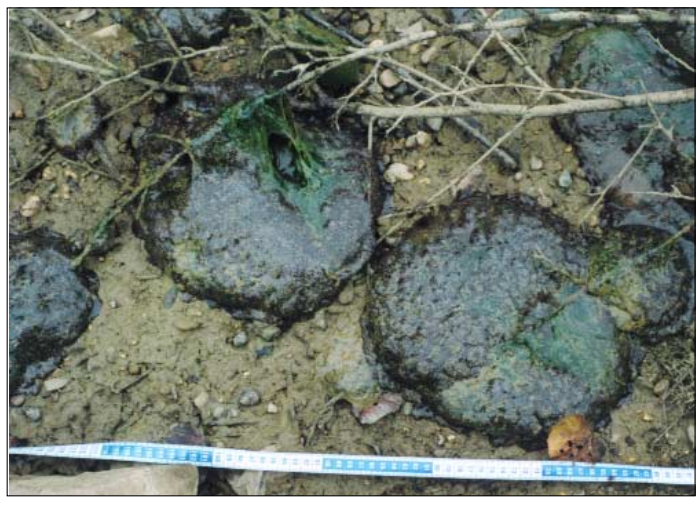

Figure 7

Colonies échouées après la vidange du canal de Haute-Saône, Canal de HauteSaône, 1998 (Territoire de Belfort - 90). Photographie de S. RODRIGUEZ.

Figure 7

Stranded colonies after the draining of the canal of Haute-Saône, canal of HauteSaône, 1998 (Territoire de Belfort - 90). Photography by S. RODRIGUEZ. 


\section{BIOLOGIE}

Pectinatella présente trois modes de reproduction : sexué, par bourgeonnement et à partir des statoblastes.

La chronologie du développement de l'espèce permet de décrire et comparer ces trois modes de reproduction.

En climat tempéré, lorsque les conditions thermiques ne sont plus propices (automne, température $<12^{\circ} \mathrm{C}$ BROWN, 1933 - basses températures défavorables EVERITT, 1975), la colonie dégénère et seules subsistent les formes hibernantes : les statoblastes.

A la mort de la colonie, les statoblastes flottent à la surface, agrégés les uns aux autres par la substance hyaline ou se trouvent très souvent agglutinés au sein des spermaphytes immergés (Saône, 1998) et des masses algales, BROWN (1933) signalant plus d'un million de statoblastes dans un amas de Spirogyra sp.

Ces statoblastes survivent à des contraintes environnementales difficiles et assurent ainsi aux colonies la continuité de vie d'années en années et de générations en générations (BRIEN, 1960).

Ainsi, les statoblastes de $P$. magnifica sont-ils encore viables après une exposition de 3 ans à basses températures - réfrigérateur (ODA, 1979), la germination des statoblastes étant seulement affectée par une température maintenue à $-12^{\circ} \mathrm{C}$ pendant 175 jours (BROWN, 1933).

La dessiccation apparaît plus limitante, la mort de tous les statoblastes pouvant intervenir en 3 jours (ODA, 1979).

La tolérance à l'eau de mer est de 6 mois selon ODA, 1990 (in SEO, 1998).

Quelques statoblastes (OSBURN, 1921) sont encore capables de germer après passage dans le tube digestif d'animaux inféodés aux milieux aquatiques (amphibiens, reptiles, canards).

Au printemps, lorsque les conditions thermiques (minimale : $9^{\circ} \mathrm{C}$, optimale : $19-27^{\circ} \mathrm{C}$, BROWN 1933) et de la photopériode deviennent favorables, les deux valves du statoblaste s'ouvrent et donnent naissance à un nouvel individu (zoécie). La colonie se forme à la faveur du bourgeonnement du cystide qui va engendrer des individus filles qui, à leur tour, bourgeonneront.

Selon BRIEN (1960), la reproduction sexuée des phylactolèmes (Bryozoa) est "l'apanage des jeunes colonies nées des statoblastes ». Les gamètes mâles et femelles sont produits, de mi-mai à juin, au sein du cystide. Après fécondation, la larve va achever son développement au sein d'un cystide clos dépourvu de polype. Après éclosion la larve nage et se fixe sur un support.

La métamorphose donne naissance à un nouvel individu qui par bourgeonnement forme une nouvelle colonie.

La formation des statoblastes intervient, au sein du cystide, lors de la phase de croissance de la colonie. Ce phénomène n'est nullement provoqué par des facteurs environnementaux défavorables (BRIEN, 1960). 


\section{ÉCOLOGIE}

\section{Habitat}

L'espèce semble assez eurytope à tendance lénitophile et atteint ses développements maximums dans les eaux dormantes ; les formes jeunes adhèrent au substrat puis flottent en pleine eau.

Deux pôles habitationnels semblent privilégiés :

- les substrats plans : pierres (RICCIARDI et LEWIS, 1991 ; LACOURT, 1968 ; ODA, 1979), coque de bateau (HYMAN, 1959), palissades en bambous et vieux pneus (ODA, 1979), coquille d'Anondonta grandis (CURRY et al., 1981) et ouvrage de maçonnerie (NOTTENGHEM, 1999) ;

- les végétaux, branchages (LACOURT, 1968), Elodea canadensis, Nymphea tuberosa, (RICCIARDI et LEWIS, 1991) et Ceratophyllum demersum (BROWN, 1933 ; RICCIARDI et LEWIS, 1991), Potamogeton sp. et Ranunculus sp. (échantillons RNB 1999, DIREN Franche-Comté et S. RODRIGUEZ).

Des expériences menées en aquarium ont précisé le choix des larves de P. magnifica (HUBSCHMAN, 1970) pour différents types de substrats :

- préférence des pierres aux surfaces de verre ;

- en présence de sable, fixation sur les vitres d'un aquarium ;

- surfaces naturelles préférées au sein de différents supports (plastique, aluminium, pierres calcaires, Typha morts).

La profondeur maximale de colonisation notée pour les observations franc-comtoises et limitrophes semble se situer au voisinage de $3 \mathrm{~m}$, l'abondance maximale étant la plupart du temps observée dans 1 à 2 m d'eau, en stations très éclairées (BROWN, 1933).

\section{Température}

L'espèce semble assez thermophile (BROWN, 1933) et prospère dans des eaux dont la température dépasse $20^{\circ} \mathrm{C}$ (BROWN, $1933: 21,5^{\circ} \mathrm{C}$; EVERITT, $1975: 22$ à $32^{\circ} \mathrm{C}$; RICCIARDI et LEWIS, $1991: 21^{\circ} \mathrm{C}$ ). Le maximum d'activité se situant au milieu de l'été en zone tempérée.

Les valeurs de températures relevées sur les sites où l'espèce a été récoltée (Saône et Ognon) d'avril à octobre se situent entre $9^{\circ}$ et $24^{\circ} \mathrm{C}$.

\section{Type et qualité de l'eau} eau :

La littérature comporte quelques valeurs de paramètres physico-chimiques de pleine

- pH 6.8 - 7.1 (EVERITT, 1975) à 8 (BROWN, 1933) ;

- conductivité comprise entre 80 et $330 \mu$ Siemens/cm (BROWN, 1933) ;

- BROWN (op. cit) signale l'effet défavorable d'un déficit en oxygène sur la germination des statoblastes ;

- EVERITT (op. cit.) qualifie le type d'eau favorable au développement de $P$. magnifica de légèrement à modérément alcaline, avec une basse conductivité et strictement dulçaquicole. 
Une série de caractéristiques physico-chimiques de pleine eau mesurée sur les sites de récoltes cités plus haut est rassemblée dans le Tableau II.

Les valeurs observées caractérisent une eau fortement minéralisée, à $\mathrm{pH}$ à tendance alcaline, riche en composés de l'azote et du phosphore et marquée par une forte activité biologique et notamment par de fortes concentrations en chlorophylles et phéopigments.

Les indices de qualité biologique globale se situent entre 10 et $17 / 20$ et soulignent l'existence d'épisodes d'altération locale de la biocénose zoomacrobenthique.

P. magnifica est qualifiée par de nombreux auteurs comme tolérante à la pollution organique et présentant une tendance à proliférer dans les eaux turbides (SMITH, 1985), compte tenu de son mode de nutrition par filtration.

\section{MODE DE COLONISATION ET RÉPARTITION MONDIALE DE P. MAGNIFICA}

La capacité des statoblastes à résister au gel et à la dessiccation a permis à l'espèce de coloniser l'hémisphère nord.

Les vecteurs de dissémination sont nombreux :

- hydrochorie (anneau pneumatique du statoblaste) ;

- zoochorie, surface du corps des mammifères et des oiseaux (ODA, 1974) et ingestion par de jeunes poissons (Micropterus salmoides, Pomoxis annularis, Lepomis pallidus, Dorosama cepedionum) et d'autres organismes inféodés au milieux aquatiques (OSBURN, 1921) ;

- anthropochorie :

* coque d'un navire transatlantique (HYMAN, 1959) ;

* transport de poissons d'aquacultures (SEO, 1998) ;

* déplacements d'équipements de pêche (SEO, 1998) ;

- anémochorie, du fait de la taille des statoblastes ce mode de dissémination peut être envisagé. (J.Y. CRETIN, communication orale).

Cette espèce, observée pour la première fois dans l'Est Américain (Philadelphie) et décrite par LEIDY (1851), a été signalée pour la première fois, en Europe, à Hambourg en 1883. En 1930, BORG évoquait son caractère cosmopolite et, en 1959, HYMAN soulignait son endémisme à l'est des Etats Unis et son introduction en Europe centrale.

En 1968, LACOURT met en doute l'introduction de cette espèce en Europe sans en apporter réellement la preuve.

En 1973, sa présence est signalée au Japon (MAWATARI, 1973) et elle fait son apparition en Corée, en 1995 (SEO, 1998).

L'espèce n'a jamais été observée, à ce jour, dans l'hémisphère sud. 
Tableau II

Caractéristiques physico-chimiques de la pleine eau.

Table II

Physicochemical features of the water.

\begin{tabular}{|c|c|c|c|c|c|c|c|c|}
\hline cours d'eau & station & date & date & $\begin{array}{c}\text { nbr de } \\
\text { mesures }\end{array}$ & Température & Conductivité & pH & DBO5 \\
\hline & & & & & ${ }^{\circ} \mathrm{C}$ & $\mu \mathrm{S} / \mathrm{cm}$ & & $\mathrm{mg} / 1$ \\
\hline Saône & Scey-sur-Saône & $12 / 05 / 98$ & $13 / 10 / 98$ & 6 & 11 à 22,2 & 303 à 530 & 7,7 à 8,9 & 2 à 5,3 \\
\hline Ognon & Beaumotte Aubertans & $26 / 06 / 99$ & $19 / 10 / 99$ & 4 & 10,3 à 23,1 & 322 à 431 & 7,9 à 8,1 & 1,4 à 2,2 \\
\hline Saône & Auxonne & $21 / 04 / 98$ & $13 / 10 / 98$ & 8 & 9 à 24 & 295 à 451 & 7,9 à 8,4 & $<0,5$ à 3,9 \\
\hline Saône & Apremont & $08 / 04 / 98$ & $13 / 10 / 98$ & 7 & 10,9 à 23 & 376 à 500 & 7,8 à 8,2 & 1,4 à 5,3 \\
\hline
\end{tabular}

\begin{tabular}{|c|c|c|c|c|c|c|c|c|c|c|c|}
\hline cours d'eau & station & date & date & $\begin{array}{c}\text { nbr de } \\
\text { mesures }\end{array}$ & Ammonium & Nitrites & Nitrates & Phosphates & PT & Chlorophylle a & Phéopigments \\
\hline & & & & & $\mathrm{mg} / \mathrm{l}$ & $\mathrm{mg} / \mathrm{l}$ & $\mathrm{mg} / \mathrm{l}$ & $\mathrm{mg} / \mathrm{l}$ & $\mathrm{mg} / \mathrm{l}$ & $\mu \mathrm{g} / \mathrm{l}$ & $\mu \mathrm{g} / \mathrm{l}$ \\
\hline Saône & Scey-sur-Saône & $12 / 05 / 98$ & $13 / 10 / 98$ & 6 & 0,03 à 0,12 & 0,07 à 0,11 & 3,89 à 7,89 & 0,3 à 0,99 & 0,14 à 0,38 & 6,68 à 43,5 & 3,51 à 15 \\
\hline Ognnon & Beaumotte Aubertans & $26 / 06 / 99$ & $19 / 10 / 99$ & 4 & 0,03 à 0,05 & 00,2 à 0,03 & 3,84 à 6,54 & 0,05 à 0,1 & 0,04 à 0,06 & 2,15 à 8,01 & 1,02 à 1,79 \\
\hline Saône & Auxonne & $21 / 04 / 98$ & $13 / 10 / 98$ & 8 & $<0,05$ à 0,11 & 0,04 à 0,08 & 2,8 à 14,1 & $<0,05$ à 0,41 & 0,77 à 8,58 & 0,28 à 3,48 \\
\hline Saône & Apremont & $08 / 04 / 98$ & $13 / 10 / 98$ & 7 & 0,01 à 0,22 & 0,04 à 0,08 & 4,93 à 14,3 & 0,23 à 0,82 & 0,1 à 0,31 & 1,61 à 36,3 & 0,03 à 15,9 \\
\hline
\end{tabular}

\begin{tabular}{|c|c|c|c|c|}
\hline cours d'eau & station & $\begin{array}{c}\text { Variété } \\
\text { faunistique }\end{array}$ & $\begin{array}{c}\text { Groupe } \\
\text { Indicateur }\end{array}$ & IBGN/20 \\
\hline Saône & Scey-sur-Saône & 29 & 5 & 13 \\
\hline Ognon & Beaumotte Aubertans & 33 & 7 & 16 \\
\hline Saône & Auxonne & 47 & 6 & 17 \\
\hline Saône & Apremont & 24 & 4 & 10 \\
\hline
\end{tabular}


A l'appui de cette dissémination, SEO souligne en 1998 le caractère adventice de cette espèce en spécifiant que la taille importante des colonies ne leur aurait pas permis, auparavant, d'échapper aux observateurs des stations extrêmes orientales.

L'espèce a été observée pour la première fois à Hambourg (1883) puis dans plusieurs localités de l'Europe de l'Est (LACOURT, 1968) par dissémination dans les corridors fluviaux de l'Elbe et de l'Oder (Tableau III et Figure 8).

\section{Tableau III}

Répartition mondiale de Pectinatella magnifica Leidy 1851.

\section{Table III}

World distribution of Pectinatella magnifica Leidy 1851.

\begin{tabular}{|c|c|c|c|c|}
\hline Date & Lieu & \begin{tabular}{|c|} 
Taille des \\
colonies \\
en $\mathrm{cm}$
\end{tabular} & Auteurs & Remarque \\
\hline 1851 & $\begin{array}{l}\text { Rivière aux environs de } \\
\text { Philadelphie }\end{array}$ & & LEIDY, 1851, in LACOURT, 1968 & espèce type \\
\hline \multirow[t]{3}{*}{1851} & $\begin{array}{l}\text { Fresh Pond et Mystic Pond, } \\
\text { (Massachusetts) }\end{array}$ & \begin{tabular}{|c|}
5 \\
(diamètre) \\
\end{tabular} & \multirow{3}{*}{ LEIDY, 1851, in JULLIEN 1885} & \\
\hline & Pennisewassee Pond (Maine) & & & \\
\hline & $\begin{array}{l}\text { Environs de Columbus } \\
\text { (Mississipi) }\end{array}$ & $38 \times 30$ & & \\
\hline 1883 & La Bille près de Hambourg & & $\begin{array}{c}\text { KRAEPELIN, 1887, in } \\
\text { SCHACHANOWSKAJA, } 1929\end{array}$ & \\
\hline 1902 & L'Havel, Spandau (Berlin ouest) & & SCHACHANOWSKAJA, 1929 & \\
\hline \multirow[t]{2}{*}{1905} & L'Oder, Breslau (Silésie) & & \multirow{2}{*}{$\begin{array}{c}\text { ZIMMER, } 1906 \text { in } \\
\text { SCHACHANOWSKAJA, } 1929\end{array}$} & \\
\hline & L'Oder (Francfort) & & & \\
\hline 1928 & Bohême & & SCHACHANOWSKAJA, 1929 & $\begin{array}{l}\text { Observe } 1 \\
\text { statoblaste }\end{array}$ \\
\hline 1931 & Rivière Huron, Michigan & & \begin{tabular}{|c|} 
BROWN, 1933 \\
\end{tabular} & \\
\hline 1937 & Bassins dans l'lowa & 60 & JUDD, 1950, in BUSHNELL, 1965 & \\
\hline 1942 & Passion river, Guatemala & & $\begin{array}{l}\text { ROGICK et BROWN, 1942, } \\
\text { in LACOURT, } 1968\end{array}$ & Statoblastes \\
\hline 1950 & Environs d'Hamilton, Ontario & $28 \times 35$ & JUDD, 1950, in BUSHNELL, 1965 & \\
\hline 1957 & Lac Egridir (Turquie) & & GELDIAY, 1957, in LACOURT & \\
\hline 1959 & $\begin{array}{l}\text { Entre l'Elbe et et l'Oder, absente } \\
\text { de Hongrie }\end{array}$ & & $\begin{array}{l}\text { SEBESTYÉN, } 1959 \\
\text { in LACOURT, } 1968\end{array}$ & \\
\hline 1962 & Lake Snagov (Roumanie) & & $\begin{array}{c}\text { CÃPUSE, 1962, } \\
\text { in LACOURT, } 1968 \\
\end{array}$ & \\
\hline 1963 & Alabama & & DENDY, 1963, in EVERITT, 1975 & \\
\hline 1973 & Japon & & MAWATARI, 1973, in SEO, 1998 & \\
\hline \multirow[t]{2}{*}{1975} & Sud-Est de la Louisiane & & \multirow[b]{2}{*}{ EVERITT, 1975} & \multirow{2}{*}{$\begin{array}{c}\text { Absence } \\
\text { d'inventaire } \\
\text { antérieur des } \\
\text { bryozoaires }\end{array}$} \\
\hline & Caddo Lake, Texas & & & \\
\hline 1994 & Franche-Comté & & RODRIGUEZ et VERGON, 1999 & \\
\hline 1995 & $\begin{array}{l}\text { A proximité de Taechungho } \\
\text { (Corée) }\end{array}$ & $\begin{array}{c}50 \\
\text { (diamètre) }\end{array}$ & SEO, 1998 & \\
\hline
\end{tabular}

en italique : correspond à l'année de publication 
En 1957 elle fait son apparition en Asie Mineure (LACOURT, 1968). La présence de $P$. magnifica ne peut être ici expliquée par le phénomène d'hydrochorie : le lac Egridir où elle est mentionnée sur le plateau anatolien étant un lac karstique avec émissaire souterrain (communication personnelle J.F. DUCROZ). Les observations de LACOURT ne précisent pas si cette espèce est endémique ou a été apportée par les oiseaux, l'alevinage ou du matériel de pêche.

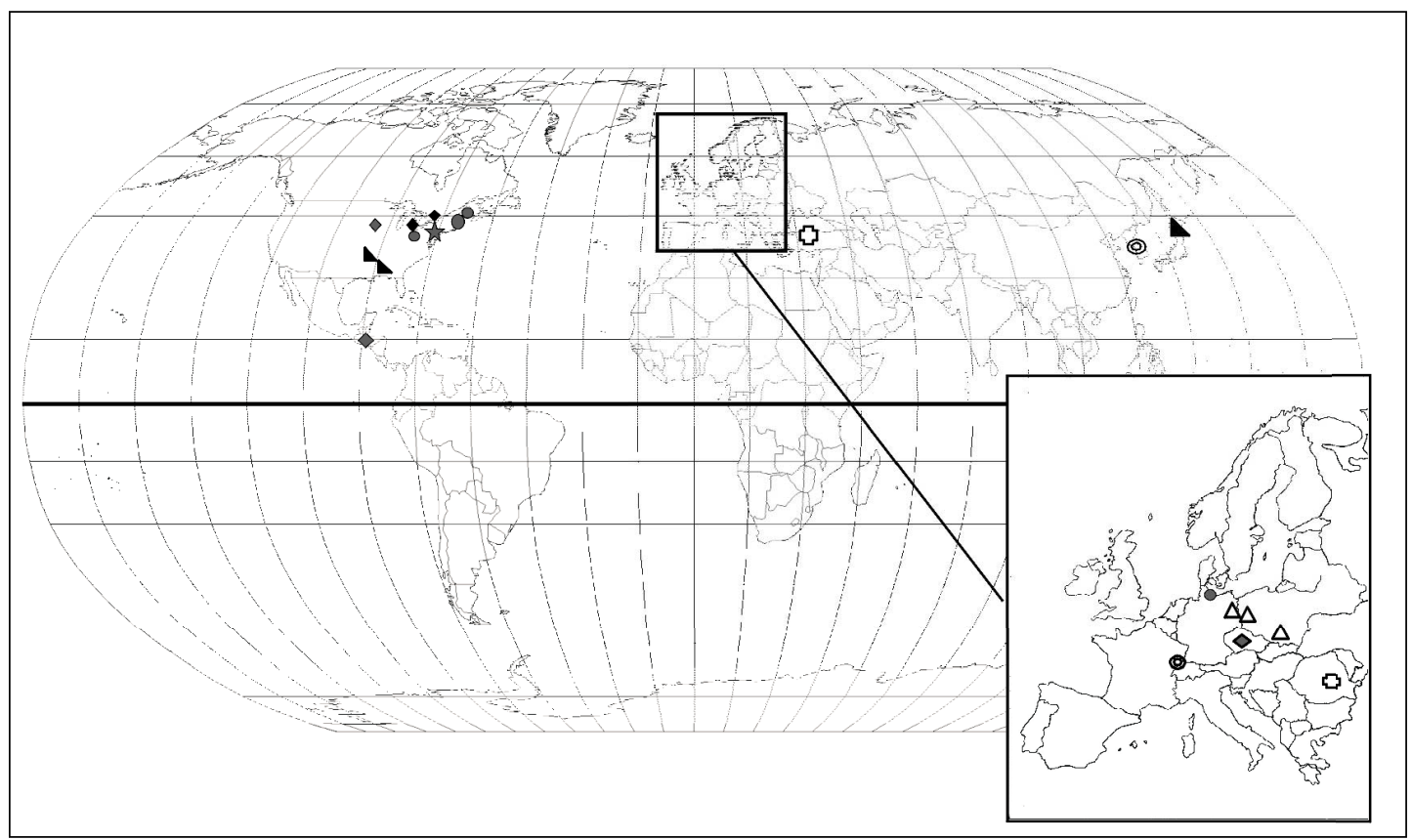

\section{Figure 8}

Répartition mondiale de Pectinatella magnifica Leidy 1851.

\section{Figure 8}

World distribution of $P$. magnifica Leidy 1851.

Plus récemment, P. magnifica a fait son apparition en France, en 1994 (RODRIGUEZ et VERGON in NOTTENGHEM, 1999) à partir du nord-est de la Franche-Comté, puis par colonisations successives des départements limitrophes (Figure 9, Tableau I). Bien qu'absente du Rhin (FRANZ, 1992), elle est très certainement parvenue sur le territoire français par le canal navigable du Rhône au Rhin. 


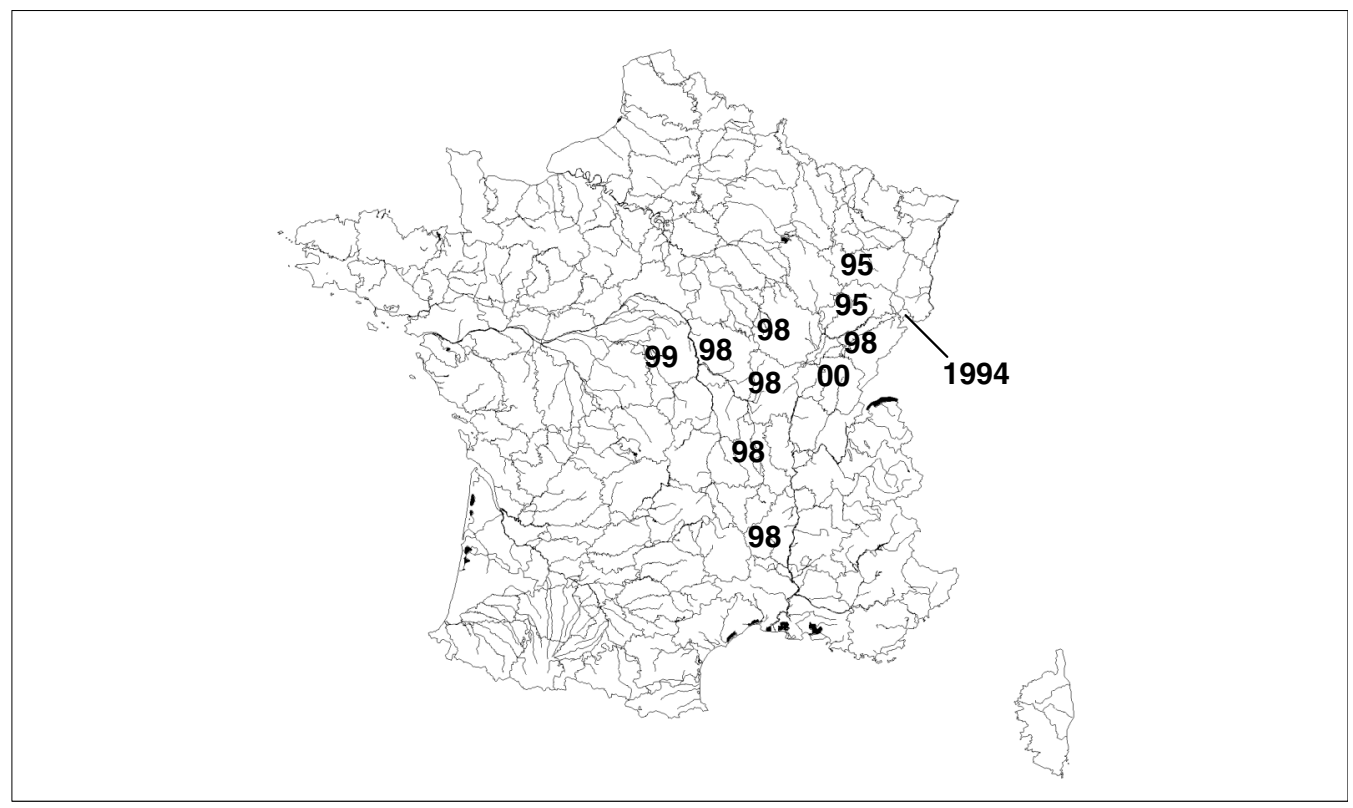

Figure 9

Carte de répartition spatio-temporelle de $P$. magnifica sur le territoire métropolitain français.

\section{Figure 9}

Space-time distribution map of $P$. magnifica on metropolitan French territory.

\section{PROBLÉMATIQUE}

L'été 1999 s'est caractérisé par des développements spectaculaires du Bryozoaire dans les plans d'eau du nord Franche-Comté, des dizaines de colonies ayant envahi jusqu'à $50 \%$ du volume d'eau de certains d'entre eux (étang des Trémeurs, 88 et étang de Lachapelle-sous-Chaux, 90).

Cette situation, marquée par l'inquiétude des propriétaires d'étangs, s'est manifestée par de nombreux appels auprès du service de l'eau et des milieux naturels (SEMNA) de la Direction Régionale de l'Environnement (DIREN de Franche-Comté). L'échec de leurs tentatives pour détruire les colonies (mise à sec, chaulage, etc.) a conduit les auteurs à engager une enquête sous forme d'une fiche signalétique (ci-jointe) faisant apparaître les cinq domaines de problématique suivants :

- bilan des connaissances acquises et répartition de l'espèce ;

- causes de la vitesse de progression de l'espèce en Franche-Comté ;

- facteurs à l'origine de la très grande taille des colonies (conditions climatiques particulières, modalités de germination, situation trophique des étangs) ;

- modes de gestion des plans d'eau favorables au développement de P. magnifica ;

- à partir de ces données, quelles techniques de limitation et de destruction des colonies appliquer (récolte manuelle, chaulage, mise a sec et évolage, incinération) ? Des essais d'attaque à l'acide de petites colonies sont en cours au laboratoire des services vétérinaires de Haute-Saône (LEHONG, communication personnelle). 
Sylvaine Rodriguez : srodrig@fr.packardbell.org Jean-Paul Vergon : jean-paul.vergon @franche-comte.environnement.gouv.fr

Fiche d'enquête « Bryozoaire : P. magnifica »

\section{Nom et coordonnées de l'observateur :}

Date de l'observation :

Date d'apparition :

Date de disparition :

\section{Description}

Taille des colonies :

Forme :

Fixée

pleine eau

Présence de forme de résistance (statoblastes)

oui-

non-

\section{Données stationnelles}

- Commune :

- Département :

- Nom du cours d'eau ou du plan d'eau :

- Bassin versant :

- Géologie du secteur :

- Particularités climatiques ayant précédé l'événement :

\section{Données physico-chimiques}

- Température de l'eau :

- Température de l'air :

- Concentration en oxygène :

- $\mathrm{pH}$ :

- Conductivité :

- Transparence :

- Autres paramètres :

\section{Observations}

- Mode de traitement éventuel (récolte, chaulage, mise à sec) et effets :

Autres remarques: 
La première étape du travail engagé a porté sur la réalisation et la diffusion de la fiche d'enquête relayée par l'Association Française de Limnologie (AFL) au niveau national.

\section{REMERCIEMENTS}

Les auteurs tiennent à remercier l'Association Française de Limnologie et toutes les personnes ayant fait retour du questionnaire et leur ayant fourni des photographies de colonies en place, des éléments d'écologie, de répartition, et d'essais de limitation des colonies.

Nous adressons également nos remerciements au Professeur Jean-Loup D’HONDT ainsi qu'à Patrice NOTTENGHEM qui ont confirmé la détermination de $P$. magnifica et nous ont permis d'accéder à certains documents.

\section{BIBLIOGRAPHIE}

ALOCAT L., 1961. Bref aperçu sur les méduses d'eau douce et découverte de l'une d'entre elles, Craspedacusta sowerbii Lankester, dans la région de Montbéliard. Bull. Soc. Hist. Nat. Doubs, 63 (2), 31-36.

BORG F., 1930. Moostierchen oder Bryozoen (Ectoprocta). In : Tierwelt Deutchlands, DAHL F. ed., 17, 25-142.

BRIEN P., 1960. Classe des Bryozoaires. In : GRASSÉ P. ed., Traité de zoologie, 5 (2), 1054-1335, Paris.

BROWN C.J.D., 1933. A limnological study of certain fresh-water Polyoza with special reference to their statoblasts. Trans. Amer. Microsc. Soc., 52 (4), 271-316.

BUSHNELL J.H., 1965. On the taxonomy and distribution of freshwater Ectoprocta in Michigan. Part I. Trans. Amer. Microsc. Soc., 84 (2), 231-244.

CURRY M.G., EVERITT B., VIDRINE M.F., 1981. Hapto benthos on shells of living clams in Louisiana U.S.A. Wassmann j. biol., 39 (1-2), 56-62.

D'HONDT J.L., CONDÉ B., 1996. Une espèce de Bryozoaires d'eau douce (Phylactolaemates) nouvelle pour le faune française : Pectinatella magnifica (Leidy, 1851). Bull. mens. Soc. Linn. Lyon, 65 (10), 322-326.

EVERITT B., 1975. Fresh-water Ectoprocta: distribution and ecology of five species in southeaster Louisiana. Trans. Amer. Microsc. Soc., 94 (1), 130-134.

FRANZ H., 1992. Der Rhein und seine Besieldung im Wandel: Schwebstoffzehrende Organismen (Hydrozoa, Kamptozoa und Bryozoa) als Indikatoren für den ökologischen Zustand eines Gewässers. Pollichia-Buch, 25, 1-167.

HUBSCHMAN J.H., 1970. Substrate discrimination in Pectinatella magnifica Leidy Bryozoa. J. Exp. Biol., 52, 603-607.

HYMAN L.B., 1959. The Invertebrates, smaller coelomates groups, vol. V. In : MC GRAVHill Book company, inc. New-York, 1-783.

JULLIEN J., 1885. Monographie des Bryozoaires d'eau douce. Bull. Soc. zool. Fr., 10, 64-68.

LACOURT A.W., 1968. A monograph of the freshwater Bryozoa-Phylactolaemata. Zool. verh., 93, 1-159.

LEIDY J., 1851. On Cristatella magnifica n. sp. Proc. Acad. nat. Sc. Philaelphia., 5, 265-266 (non consulté).

MAWATARI S., 1973. New occurrence of Pectinatella magnifica (Leidy) in a japanes lake. Proc. Jap. Soc. Syst. Zool., 9 m, 41-43 (non consulté).

NOTTENGHEM P., 1999. Pectinatella magnifica (Leidy, 1851), une nouvelle espèce de Bryozoaires pour la Bourgogne. La Physiophyle, 131, 12-25.

ODA S., 1974. Pectinatella magnifica occurring in Lake Shoji, Japan. Proc. Jap. Soc. syst. Zool., 10, 31-39. 
ODA S., 1979. Germination of the statoblasts of Pectinatella magnifica, a freshwater Bryzoan. In : LARWOOD G.P., ABBOTT M.B. Eds. : Advances in Bryozoology, Acad. Press, London, New-York, San Francisco, 93-112.

OSBURN R.C., 1921. Bryozoa as food for other animals. Science, 53 (1376), 451-453.

PRENANT M., BOBIN G., 1956. Bryozoaires, première partie: Ectoproctes, Phylactolaemates, Cténostomes. Faune de France, LECHEVALIER ed. Paris, 60, 1-398.

RICCIARDI A., LEWIS D.J., 1991. Occurrence and ecology of Lophopodella carteri (Hyatt) and other freswater Bryozoa in the lower Ottawa River near Montreal, Quebec. Can j. zool., 65 (5), 1401-1404.

RODRIGUEZ S., VERGON J.P., 1999. Fiche d'enquête Bryozoaires : Pectinatella magnifica. DIREN Franche-Comté ed. Besançon, 1-3

SCHACHANOWSKAJA J., 1929. Pectinatella magnifica Leidy in Böhmen. Zool. Anz., 80, 296-301.

SEO J.E., 1998. Taxonomy of the freshwater Bryozoans from Korea. Korean J. Syst. Zool., 14 (4), 371-381.

SMITH D.G., 1985. Lophopodella carteri (Hyatt), Pottsiella erecta (Potts) and other freshwater ectoprocta in the Connecticut river (New England USA). Ohio j. sci., 85 (1), 67-70.

TACHET H., BOURNAUD M., RICHOUX P., 1991. Introduction à l'étude des macroinvertébrés d'eau douce. A.F.L. Lyon, $4^{\text {ème }}$ édition, 1-155. 